\title{
X-ray Performance Evaluation of the Dexela CMOS APS X-ray Detector Using Monochromatic Synchrotron Radiation in the Mammographic Energy Range
}

\begin{abstract}
Digital detectors based on complementary metal-oxide-semiconductors (CMOS) active pixel sensor (APS) technology have been introduced recently in many scientific applications. This work is focused on the X-ray performance evaluation of a novel CMOS APS detector in low energy medical imaging applications using monochromatic synchrotron radiation (i.e., 17-35 $\mathrm{keV}$ ), which also allows studying how the performance varies with energy. The CMOS sensor was coupled to a Thallium-activated structured cesium iodide (CsI:Tl) scintillator and the detector's X-ray performance evaluation was carried out in terms of sensitivity, presampling modulation transfer function (pMTF), normalized noise power spectrum (NNPS) and the resulting detective quantum efficiency (DQE). A Monte Carlo simulation was used to validate the experimentally measured low frequency DQE. Finally, the effect of iodine's secondary generated K-fluorescence X-rays on pMTF and DQE results was evaluated. Good agreement (within 5\%) was observed between the Monte Carlo and experimentally measured low frequency DQE results. A CMOS APS detector was characterized for the first time over a wide range of low energies covering the mammographic spectra. The detector's performance is limited mainly by the detectability of the scintillator. Finally, we show that the current data could be used to calculate the detector's PMTF, NNPS and DQE for any mammographic spectral shape within the investigated energies.
\end{abstract}

\section{INTRODUCTION}

DIGITAL X-ray detectors based on complementary metal-oxide-semiconductor (CMOS) active pixel sensor (APS) technology have been introduced in the early 2000s in medical imaging applications [1] , [2]. The term "active", compared to "passive", is used to indicate the presence of at least one source follower transistor in each pixel, which buffers and/or amplifies the accumulated signal [ $\underline{3}$ ]. This leads to an improved speed and signal-to-noise ratio (SNR), due to the decreased read noise. Since the early 1990s charge coupled devices (CCD) and flat panel detectors (FPD, also referred to as active matrix flat panel imagers) have been used extensively in medical imaging applications []], []], [్], []]. CCD detectors have high sensitivity due to high fill factor (almost 100\%) and quantum efficiency, leading to small (down to $25 \mu \mathrm{m}$ ) pixel size (increasing the spatial resolution capability) []․ Also, CCDs demonstrate very small read noise [around 10-20 electrons $\left(\mathrm{e}^{-}\right.$) root mean square (r.m.s.)] because the charge transfer does not introduce any temporal noise. They also demonstrate marginal pixel-to-pixel and column-tocolumn fixed pattern (or structure) noise. Finally, they exhibit wide dynamic range [usually 6570 decibels $(\mathrm{dB})$ ], linear response, and high image quality in terms of SNR []ㅡ, [9]. However, the production cost of CCDs is high, which limits their active area to $2-5 \mathrm{~cm}^{2}$. Usually optical lenses, fiber optic plate (FOP) or electro-optic coupling are used to demagnify the light signal and allow coverage of the required X-ray field size in the patient. However, this demagnification stage increases the chance of a secondary quantum sink (arising from a lack of gain at a given conversion stage) to keep image quality within acceptable levels [10]. In other words, a number of optical photons can be lost in the demagnification stage. Moreover, CCDs are serial devices, i.e., the entire signal needs to pass through the same sense node before being read out. This leads to high read noise at high frame rates, limiting the use of CCDs in applications such as computed tomography (CT), tomosynthesis or fluoroscopy [11]. It should be noted that at low dose levels in fluoroscopy, image intensifier based CCDs are commonly used to enhance image quality by multiplying secondary generated electrons. However, these devices suffer from veiling glare caused by the electrons spread inside the image intensifier tube and visible light photons being scattered on the output optics degrading image contrast [6], [12]. Moreover, electron trajectories inside the tube can be distorted by the earth's magnetic field leading to S- 
shape distortions in the image [13]. Pincushion and Barrel-type distortions occurring due to inherent limitations of the electron focusing optics can influence the anatomy shape and lead to misdiagnosis in some cases [1ㅡ]. Finally, CCDs require high power [ㅇ] and are susceptible to radiation damage [14].

To overcome the CCD limitations, FPDs have become recently the detector of choice in medical imaging applications. FPDs are high-performing, radiation hard and large area (up to $43 \mathrm{~cm} \times 43$ $\mathrm{cm}$ for general radiography applications) detectors. However, FPDs have reduced detective quantum efficiency (DQE) at low exposure levels [15], [16], caused by high read noise due to the use of passive pixels [17], [18]. Finally, FPDs at high frame rates show an excess of image lag, ghosting and baseline drifts due to the amorphous structure of silicon ( $\mathrm{Si}$ ) or selenium (Se) [19], [20]. CMOS sensors offer an alternative to CCDs and FPDs due to the potential of radiation tolerance, low-cost mass production, low power consumption [21], and very fast image acquisition due to random pixel addressing capability [22]. Stitching and tilling technologies can be used to obtain large area sensors [18]. Additionally, CMOS sensors demonstrate low read noise at high frame rates due to column parallel read out [23] . The pixel pitch of CMOS sensors $(50-100 \mu \mathrm{m})$ is smaller compared to that of FPDs $(100-130 \mu \mathrm{m})$. The fill factor of a typical CMOS APS is around $75-80 \%$ because each pixel contains at least three transistors. The limited fill factor, combined sometimes with modest quantum efficiency, may result in decreased X-ray sensitivity compared to CCDs. However, the relatively low read noise of CMOS APS (around $100-300 \mathrm{e}^{-}$r.m.s.) usually results in high DQE values at low exposure levels. Finally, CMOS sensors suffer from pixel-to-pixel and column-to-column gain variations (or fixed pattern noise) but gain correction (based on flat fielding) can be used to compensate for this. It should be noted that researchers have recently moved towards combining the low read noise, high charge generation, and collection performance of CCDs with fast readout, low power consumption, and high integration capability of the CMOS [23]. In a hybrid CCD/CMOS array, the CCD pixels are connected to a CMOS readout integrated circuit and the column parallel readout architecture overcomes the speed versus noise limitations of a conventional CCD. Nowadays, hybrid CMOS detectors have a limited area (up to around $3 \mathrm{~cm} \times 3 \mathrm{~cm}$ ) and are used in scientific applications. For instance, hybrid CMOS X-ray detectors have been developed recently for future space-based X-ray telescope missions [24],$[\underline{25}]$.

A CMOS-based X-ray detector is an indirect conversion system. In particular, it consists of a CMOS sensor (back-end), which is sensitive to visible light photons, optically coupled to a scintillator (front-end) which converts the X-rays to secondary information carriers, i.e., optical photons [6]. The light signal produced by the scintillators increases as a function of the absorbed X-ray energy, leading to energy integrating X-ray detectors. Photon counting detectors, based on cadmium telluride (CdTe) or cadmium zinc telluride (CZT), can be used for medical imaging [26]. They offer high absorption efficiency, spatial resolution, and increased sensitivity because they are direct conversion detectors (i.e., the X-rays are converted directly into electric charge). However, the detector arrays are usually quite small (around $2 \mathrm{~cm}^{2}$ ) and scanning is required for imaging larger objects, resulting in image blurring along the scanning direction. Additionally, long acquisition times may lead to patient discomfort and movement, resulting in further image blurring.

Thallium-activated structured cesium iodide (CsI: Tl) scintillators are widely used in low energy medical imaging applications because they offer high spatial resolution and increased X-ray detectability due to the K-absorption edges of iodine (I) at $33.2 \mathrm{keV}$ and cesium (Cs) at $36.0 \mathrm{keV}$. It should be noted that X-ray detectors can be either front- or back-side illuminated. In the latter case, the light photons fall on the back-side of the chip preventing loss due to reflections on the metal gates, resulting in almost $100 \%$ fill factor and a substantial increase in quantum efficiency. However, it is difficult to manufacture such detectors because they require wafers 
uniformly and precisely thinned down to $30 \mu \mathrm{m}$ or less [27]. Digital X-ray detectors open the way to advances in the state-of-the-art of mammography, such as contrast enhanced digital mammography [28], [299], breast tomosynthesis [30], [31], cone beam CT [32], and others. An important requirement in all cases is the high detectability of X-rays at low dose levels, because for all these methods a sequence of images is acquired. In the current study we used monochromatic synchrotron radiation to measure the X-ray performance of a CMOS APS X-ray detector as a function of energy in terms of X-ray sensitivity [or signal transfer property (STP)], presampling modulation transfer function (pMTF), normalized noise power spectrum (NNPS), and the resulting detective quantum efficiency (DQE). A previously validated Monte Carlo technique [33], [34], [35] was also used to compare the maximum X-ray detectability of the system to the experimentally measured DQE at low spatial frequencies. It is worth mentioning that during the last decade several researchers used synchrotron monochromatic radiation to investigate the performance of CMOS-based X-ray detectors for small-angle X-ray scattering,

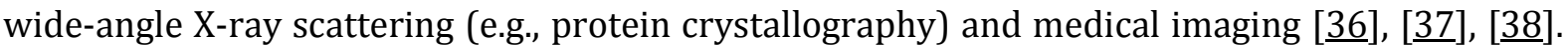
The choice of these detectors was based mainly on the large area, suitable pixel pitch, wide dynamic range, and fast readout speed of the CMOS sensors. The choice of the current detector (namely Dexela 2923 CMOS X-ray detector) for the characterization in monochromatic synchrotron radiation was driven by the detector size $(29 \mathrm{~cm} \times 23 \mathrm{~cm})$ which is suitable for mammography and breast tomosynthesis, the simplicity of the manufacturing technology, and high X-ray performance in terms of pMTF and DQE [39]. Finally, besides casting light on the sensor/scintillator behavior at different X-ray energies (notably around the iodine K-absorption edge), we also show how these data could be used to estimate the detector behavior for any Xray spectrum within the considered energy range. Hence, image simulation based on the monochromatic performance parameters can be used to predict how the mammographic image quality changes as a function of the used radiographic beam quality and exposure.

\section{MATERIALS AND METHODS}

\section{A. The CMOS APS Sensor}

The X-ray detector is based on a CMOS APS sensor developed by Dexela Limited (a PerkinElmer company). Each pixel contains an option for switching the full well capacity between two separate modes, namely high full well (HFW) with dynamic range of 73 decibels (dB) and low full well (LFW) with $69 \mathrm{~dB}$ [39], which is operated globally across the whole active area. The LFW mode has lower read noise [around 165 electrons $\left(\mathrm{e}^{-}\right)$root mean square (r.m.s.)] at the expense of reduced full well capacity (around $0.5 \times 10^{6} \mathrm{e}^{-}$) compared to the HFW mode (around $360 \mathrm{e}^{-}$r.m.s. and $1.6 \times 10^{6} \mathrm{e}^{-}$, respectively) [잉. Preliminary results demonstrated that the dark current at around $30^{\circ} \mathrm{C}$ is $6 \pm 1$ and $3 \pm 1 \mathrm{pA} / \mathrm{cm}^{2}$ in HFW and LFW modes, respectively [40]. The typical active area of a single sensor module at full resolution (pixel pitch equal to $75 \mu \mathrm{m}$ with fill factor $84 \%$ ) is $1944 \times 1536$ pixels, i.e., $14.5 \times 11.5 \mathrm{~cm}$ (namely Dexela 1512 CMOS X-ray detector). The vertical dimension consists of 6 stitched periphery blocks. Each block is connected to a dedicated 14-bit analog-to-digital converter (ADC) used to convert $\mathrm{e}^{-}$to digital numbers (DN). In the current study a $2 \times 2$ array of sensors was implemented using the tiling technology with an overall area of $29 \mathrm{~cm} \times 23 \mathrm{~cm}$ (namely Dexela 2923 CMOS X-ray detector). The average conversion gain $\mathrm{K}\left(\mathrm{e}^{-} / \mathrm{DN}\right)$ of the four sensors forming the $\mathrm{X}$-ray detector is $119.6 \pm 2.9$ and $38.8 \pm 0.6 \mathrm{e}^{-} / \mathrm{DN}$ in HFW and LFW modes, respectively [39]. The CMOS APS sensor is front-side illuminated and was optically coupled to a scintillator using a 3 $\mathrm{mm}$ thick fiber optic plate (FOP) to eliminate direct interaction of X-rays in the sensor material. It was calculated that this FOP absorbed more than $99.999 \%$ of the input X-rays in the investigated energy range. 


\section{B. Scintillator Coupled to the CMOS APS}

In this study a $200 \mu \mathrm{m}$ thick structured CsI: Tl scintillator was coupled to the CMOS APS sensor. The term "structured" refers to the high-density fibres of this scintillator, i.e., it is columnar structure (5-10 $\mathrm{mm}$ diameter) resulting from growth on a specially designed substrate [41]. This setup results in high (85\%) packing density (active phosphor volume/total screen volume) values [르. Hence, the respective coating thickness is $76.7 \mathrm{mg} / \mathrm{cm}^{2}$. The columnar structure also reduces the lateral spread of the visible photons, resulting in superior spatial resolution compared to bulk or granular scintillators. Therefore, it preserves good spatial resolution at the increased layer thickness required to have sufficient X-ray detection efficiency [43]. This scintillator features high light yield (around 55000 optical photons/MeV) and green light spectrum (with strong peak emission in the green region of the spectrum at 560 $\mathrm{nm})[\underline{44}]$.

\section{Calculation of the Maximum X-ray Detectability}

An important parameter of the scintillator is the ability to convert the input X-ray signal into useful visible light (or optical) quanta. This is described analytically by the quantum detection efficiency (QDE) and energy absorption efficiency (EAE) parameters. The QDE (also termed intrinsic efficiency) corresponds to the ratio of the absorbed over the incident number of X-ray photons. This parameter depends on the attenuation coefficient and the thickness of the scintillator. For the monochromatic X-ray beam used in the current study, the QDE is given by the following formula []ㅡ, [모]:

$$
Q D E=1-e^{-\mu_{t \sigma o t}(E) w_{0}}
$$

where $\mu_{\text {tot }}(E)$ and $w_{0}$ are the scintillator's linear attenuation coefficient [ $\underline{46}$ ] and thickness, respectively. However, scintillators are energy integrating detectors, therefore the EAE parameter is considered more appropriate as it describes the fraction of incident energy absorbed locally at the points of X-ray interaction within the scintillator [45]. This parameter depends on the amount of energy absorbed in the scintillator per absorbed X-ray photon, the Xray photons attenuated in the scintillator and the amount of incident energy. The monochromatic EAE is given by the following equation, a simplification of the integral EAE equation given in [ㅍ5]:

$$
E A E=\left(\frac{\mu_{\text {tot, }, E n}(E)}{\mu_{\text {tot }}(E)}\right)\left(1-e^{-\mu_{\text {tot }}(E) w_{0}}\right)
$$

where ${ }^{\mu_{\text {tot, }, e n}(E)}$ is the energy absorption coefficient of the scintillator [46]. This parameter includes all mechanisms of energy deposition locally at the point of X-ray interaction within the scintillator. The parameter $\mu_{\text {tot,en }}(E)$ describes the probability per unit length that the energy deposited per incident X-ray photon is locally absorbed in the scintillator, while $\mu_{\text {tot }}(E)$ represents the probability per unit length that the incident photon interacts with the material. It should be noted that when calculations of QDE or EAE are made, the packing density value needs to be considered [ $[\underline{6}$.

As mentioned above, the EAE is expected to provide a suitable representation of the signal detection efficiency, i.e., the detective quantum efficiency at zero spatial frequency [DQE(0)] [45]. This is valid for energies lower than the K-edge of the material the scintillator is made of. However, for higher energies the EAE can underestimate the X-ray detectability, because $\mu_{\text {tot, en }}(E)$ assumes that the energy of any generated K-fluorescence photon escapes the scintillator, while in this energy range $\mu_{\text {tot }}(E)$ represents the increased probability that 
both the incident and $\mathrm{K}$-fluorescence X-ray photons will be attenuated. Therefore, their ratio drops at the K-absorption edges. On the other hand, the QDE overestimates the detectability of the input signal, because it assumes that all the K-fluorescence photons are re-absorbed within the scintillator. In truth, an amount of K-fluorescence energy is re-absorbed within the scintillator, which cannot be precisely described by the analytical QDE and EAE parameters.

A Monte Carlo technique was used in this study to give a more precise description of the signal detection efficiency by calculating the actual ratio of the reabsorbed K-fluorescence X-ray photons. Monte Carlo simulations were performed considering a relative standard deviation of less than $5 \%$. X-ray detection evaluation was carried out by using a custom Monte Carlo code [33] developed for the assessment of the overall performance of indirect phosphor-based medical imaging detectors. Monte Carlo modeling has been performed for traditional rare-earth materials [33], [녕 (e.g., $\mathrm{Gd}_{2} \mathrm{O}_{2} \mathrm{~S}: \mathrm{Tb}$ ) and for compact powder phosphors under development [34] (e.g., $\mathrm{Lit}_{2} \mathrm{O}_{3}: \mathrm{Et}$ ), taking into account the principal X-ray interactions, such as: 1) photoelectric absorption (including the simulation of K-fluorescence radiation), 2) Compton, and 3) Rayleigh scattering. The validity of the code has been verified in terms of the sampling algorithm subroutines [프] and previous experimental data and analytical calculations [33], [34], [35]. Recently, a sophisticated investigation was reported in zero-frequency DQE overestimations of commercially available X-ray imaging converters (e.g., $\mathrm{Gd}_{2} \mathrm{O}_{2} \mathrm{~S}$ : Tb, $\mathrm{CsI}$ : Tl, and a-Se) based on the study of energy impartation events [35]. This study illustrates that the Monte Carlo techniques have an advantage over other theoretical approaches for the prediction of QDE and DQE(0). It should be noted that, while in principle this would have been possible also by means of established Monte Carlo packages (e.g. Geant4, MCNP, PENELOPE, FLUKA, etc.), this would have required code modifications, which would have been time consuming for the purposes of the present study.Briefly, the large area limit of the DQE (DQE(0)) for a

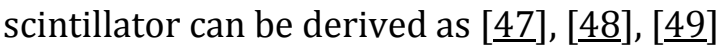

$D Q E(0)=Q D E \cdot I$

where $I$ is the Swank factor that describes the noise associated with the X-ray to light conversion process and the statistical distribution of the number of the secondary generated optical photons. For a monochromatic X-ray beam with energy $E_{i}^{0}$, the Swank factor is

calculated as $I\left(E_{i}\right)=\frac{M_{1}^{2}\left(E_{i}\right)}{M_{0}^{2}\left(E_{i}^{*}\right) M_{2}^{2}\left(E_{i}^{*}\right)}$

where $M_{j}\left(E_{i}\right)$ is the corresponding $j$ th moment of the light pulse height statistical distribution which can be measured using pulse height spectroscopy [49], [50]. Swank showed that for monochromatic X-rays and under certain reasonable conditions [47]], I is equal to the product of ${ }^{I_{A E D}}$ and ${ }^{\mathrm{I}} \mathrm{OPD}$. In particular, ${ }_{\mathrm{A}} \mathrm{AED}$ describes the statistical factor of the absorbed X-ray energy distribution as a function of energy. It depends mainly on the K-fluorescence X-rays escape and re-absorption, and slightly on X-rays undergoing inelastic effects followed by photon escape. Hence, it is close to unity for energies lower than the K-absorption edge, and drops to a minimum just above the K-absorption edge due to the escape of characteristic K-fluorescence

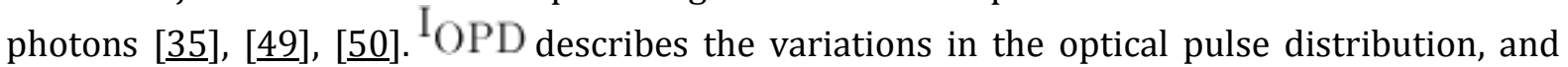
depends on the light propagation (scattering and re-absorption) inside the scintillator [마]. It is independent of the X-ray energy and depends on the scintillator material and thickness. Reference [499] determined experimentally the ${ }^{\mathrm{I}}$ OPD for different thicknesses of CsI: $\mathrm{Tl}$ scintillator, by combining the measured I with the calculated ${ }^{\mathrm{I}} \mathrm{AED}$. In the current study, the 
Monte Carlo calculated QDE and ${ }^{\mathrm{I}} \mathrm{AED}$ where combined with the bibliographic ${ }^{\mathrm{I}} \mathrm{OPD}$ to get the $\mathrm{DQE}(0)$ of the scintillator.

\section{Experimental Setup}

The X-ray performance evaluation of the detector was carried out at the SYRMEP (SYnchrotron Radiation for MEdical Physics) beamline at the ELETTRA synchrotron radiation facility in Trieste, Italy. The X-ray beam originates from one of the bending magnets of the storage ring. A double-crystal $\mathrm{Si}(111)$ monochromator is used to select the required energy in the range 8.5-35 $\mathrm{keV}$, with an energy resolution of about $10^{-3}[\underline{51}],[\underline{52}],[\underline{53}]$. In the current study, eight monochromatic energies were used for the detector evaluation: 17, 20, 23, 26, 29, 33, 33.3 and $35 \mathrm{keV}$. An ionization chamber was positioned in the "experimental" hutch at around $23 \mathrm{~m}$ from the source, to monitor the exposure. In our study, the detector was mounted on a motorized translation stage at a distance of around $32 \mathrm{~m}$ from the source in the "patient" room (the SYRMEP beamline features two hutches that can be used for imaging-an "experimental" hutch upstream and a "patient" room immediately downstream; the name of the latter comes from the in vivo program currently underway in that room [ $\underline{54}]$ ). A tungsten (W) slit system determined the cross section of the beam impinging on the detector, and this was adjusted to $206 \mathrm{~mm} \times 3$ $\mathrm{mm}$ on the detector surface. A motorized stage enabled vertical continuous scanning of the detector in the plane perpendicular to the direction of the X-ray beam with a speed of $15 \mathrm{~mm} / \mathrm{s}$. The integration time of the detector was set to $15 \mathrm{~s}$, leading to a total uniformly irradiated area of about $206 \mathrm{~mm} \times 220 \mathrm{~mm}$.

\section{E. X-ray Performance Evaluation of the Detector}

The X-ray performance of the detector was evaluated in terms of STP, pMTF, NNPS, and DQE. The STP describes the X-ray sensitivity and relates the average output signal (in DN) with the input Air Kerma ( $\mathrm{K}_{\mathrm{a} ;}$; in $\mu \mathrm{Gy}$ ) at detector surface. The average DN was calculated from a region of interest (ROI) of $2350 \times 2350$ pixels $(176.3 \mathrm{~mm} \times 176.3 \mathrm{~mm})$ from each frame over 4 frames. The images were dark field subtracted and flat field corrected to apply gain and offset correction [ㅁ5].

The pMTF describes the contrast reduction at the different spatial frequencies that compose the image, and is used to quantify the spatial resolution of an imaging system. For the determination of the pMTF, the edge technique [56] was used according to the IEC standard [ $\underline{57}$ ] based on the algorithm presented in [다] . Briefly, an opaque and polished edge test object (W foil, $1 \mathrm{~mm}$ thick, 99.95\% pure [두]) was placed at a shallow angle $\alpha\left(1.5-3^{\circ}\right)$ with respect to the detector pixel rows and columns. The pixel values of seven consecutive lines centrally located across the edge were then used to generate seven oversampled edge profiles (or edge spread function (ESF) curves) along the sampling direction. This number of ESF curves sufficiently reduces the statistical noise and simultaneously covers both dark and white areas of the edge test image. The ESF curves were shifted laterally to overlap with each other and combined to calculate the average oversampled ESF curve. The latter was then differentiated to get the oversampled line spread function (LSF). The pMTF was obtained from the modulus of the Fourier transform (FT) of the oversampled LSF and normalized to one at zero spatial frequency:

$p M T F(x)=|F T\{\operatorname{LSF}(x)\}|=\left|F T\left\{\frac{d}{d x}[E S F(x)]\right\}\right|$ 
Finally, the vertical and horizontal pMTF values were calculated (from 0.5 line pairs per millimeter $(\mathrm{lp} / \mathrm{mm})$ to Nyquist Frequency $\left(\mathrm{F}_{\mathrm{Nyq}}\right)$ with an interval of $0.5 \mathrm{lp} / \mathrm{mm}$ ) and combined to determine the average (over the edge's orientations) pMTF.

The NPS describes the spectral decomposition of the noise variance in an image as a function of spatial frequency and it expresses the noise transfer. Hence, flat field images (including background illumination only) were acquired to calculate the combined detector (also termed read), dark current- and background-induced (also termed quantum) noise. Offset and gain corrections were applied to the flat images to remove the dark offset and minimize gain variations between different pixels (also known as fixed pattern noise). A modified gain correction algorithm was used to compensate for the number of used reference flat frames [55]. The NPS was then calculated by applying a 2-D algorithm to a corrected flat field image [57], [무]

$$
N P S(u, v)=\frac{\Delta x \cdot \Delta y}{M \cdot N_{x} \cdot N_{y}} \sum_{m=1}^{M}\left|F T\left\{I\left(x_{i}, y_{i}\right)-S\left(x_{i}, y_{i}\right)\right\}\right|^{2}
$$

$I(x, y)$ is the corrected flat field image, $S(x, y)$ is a second order polynomial fit to remove low frequency (less than $1 \mathrm{lp} / \mathrm{mm}$ ) background trends, $u$ and $v$ are the spatial frequencies corresponding to $x$ and $y$ orientations, $\Delta_{x}$ and $\Delta_{y}$ are the $x$ and $y$ pixel pitches, $N_{x}$ and $N_{y}$ express the ROI size in pixels (256 pixels in our case) and $M$ is the number of ROIs used to calculate the average NPS. In order to use the NPS for the DQE calculation, seven lines on either side of the central axes of the 2-D NPS (omitting the axes themselves) were used to compute the 1-D horizontal and vertical NPS. The axes were omitted because they may be susceptible to any remnant column- or row-wise fixed pattern noise on the flat field images. The data were binned in spatial frequencies from $0.5 \mathrm{lp} / \mathrm{mm}$ to $\mathrm{F}_{\mathrm{Nyq}}$ with an interval of $0.5 \mathrm{lp} / \mathrm{mm}$ [57]. The horizontal and vertical NPS(x) were divided by the square of the mean pixel value $\left(\mathrm{DN}^{2}\right)$ of the corrected flat images (for given energy and $\mathrm{K}_{\mathrm{a}}$ ) to obtain the NNPS [으]. The mean DN corresponds to the Fourier Transform of the flat field image at zero spatial frequency and this normalization is made in accordance to the normalization of the pMTF. Finally, the horizontal and vertical 1-D NNPS values were combined (i.e., simply averaged) to calculate the average 1-D NNPS.

The DQE expresses the fraction of input X-ray quanta effectively used for image signal-to-noise ratio (SNR) at each spatial frequency, and describes the ability of a particular system to use the available input quanta. In our case, it depends on the X-ray absorption, noise, and resolution of the front-end (scintillator), and the noise and resolution of the back-end (CMOS sensor) part of the X-ray detector. It is calculated using the following formula [ㄷ]], [무]:

$D Q E(f)=\frac{S N R_{\text {out }}^{2}}{S N R_{\text {in }}^{2}}=\frac{p M T F^{2}(f)}{\frac{\Phi}{K_{a}} \cdot K_{a} \cdot N N P S(f)}$

where $\Phi / K_{a}$ is the photon fluence per exposure ratio (in X-rays per $\mathrm{mm}^{2}$ per $\mu \mathrm{Gy}$ ), assuming that the detector behaves as an ideal photon counter [61], [62], $p M T F(f)$ is the average presampling modulation transfer function and $N N P S(f)$ is the average normalized noise power spectrum. It should be noted that the current system consists of an energy integrating detector, so an energy-weighted calculation of the $\Phi / K_{a}$ would be more realistic.However, Samei and Flynn [63] demonstrated that for $70 \mathrm{kV}$ (W/Al anode/filtration combination) with additional $19 \mathrm{~mm} \mathrm{Al}$, the difference between the energy-weighed and photon-counting 
approximations is less than 3\%. This difference is even smaller for lower energy spectra. In practice, the input signal-to-noise ratio square $\left(S N R_{\text {in }}^{2}\right)$ corresponds to the photons fluence $(\Phi)$ due to the Poisson distribution of the input quanta. The output $\operatorname{SNR}^{2}\left(S N R_{\text {out }}^{2}\right)$ is calculated from the ratio between $p M T F^{2}(f)$ and $N N P S(f)$. Usually the experimentally measured $\mathrm{DQE}(0)$ is excluded from the DQE analysis because low-frequency artifacts (such as background trends) can result in unusually high NNPS at zero spatial frequency, leading to underestimation of the DQE. Therefore, its inclusion might make it hard to distinguish between low frequency artifacts and stochastic noise. As aforementioned, detrending can be used to reduce the low frequency trends. However, this does not change the mean value (i.e., the central point on the 2-D NNPS array), and it does not completely remove the excessively large values along the $u$ and $v$ axes [무] .

It should be noted that we have previously characterized the same detector under clinical mammographic (W/Al at $28 \mathrm{kV}$ ) and general radiography [W/Al at $50 \mathrm{kV}$ (RQA3) and $70 \mathrm{kV}$ (RQA5)] conditions [여․ Briefly, the mammographic DQE at $0.5 \mathrm{lp} / \mathrm{mm}$ spatial frequency [DQE(0.5)] was found in the range 0.58-0.71 in HFW mode and 0.53-0.70 in LFW mode, respectively. This study presents the X-ray performance of the detector over a wide range of mammographic energies and can form the basis for predicting the PMTF, NNPS, DQE and image quality for different mammographic beam qualities. For brevity, we demonstrate the detector's performance in HFW operation mode only.

\section{RESULTS AND DISCUSSION}

\section{A. Importance of the Investigated Parameters}

The purpose of medical imaging is to provide sufficient information about specific aspects of human body structure or function. Hence, the quality of a radiograph needs to be adequate to provide the required information for a given task. The primary physical parameters that affect image quality are spatial resolution (the ability to represent distinct anatomic features inside the irradiated object), noise (systematic and random variations superimposed on the actual measured signal), and contrast (magnitude of the relative signal difference between the object of interest and the surrounding background) [64]. The pMTF is the combination of contrast and resolution, the NPS combines the noise and resolution, and the SNR expresses the ratio between signal and noise in large scale objects (i.e., at zero spatial frequency). The combination of SNR, pMTF and NPS determines the DQE which represents the ability to visualize object details of a certain size and contrast (contrast-detail resolution). The experimentally measured X-ray performance parameters in this study are STP (which expresses the X-ray sensitivity of the detector), pMTF, NNPS, and DQE. Finally, QDE, EAE and Monte Carlo DQE(0) were calculated to express the absorption of X-rays (at zero spatial frequency) from the scintillator.

\section{B. X-ray Sensitivity of the Detector}

Fig. 1(a) shows the STP curves of the detector operated in HFW mode for different energies. It should be noted that DN refers to analog to digital conversion and therefore is an arbitrary unit. The sensor conversion gain $\left(\mathrm{K}\left(\mathrm{e}^{-} / \mathrm{DN}\right)\right)$ could be used to express the output signal in absolute units of electrons. However, in X-ray performance evaluation studies the X-ray sensitivity is commonly expressed by the output pixel value (in DN) as a function of the input $\mathrm{K}_{\text {a }}$ (in $\mu \mathrm{Gy}$ ). The detector demonstrated linear signal transfer with coefficients of determination $\left(\mathrm{R}^{2}\right)$ greater than 0.9997 in all cases. We observe an increase in the signal from the detector per unit $K_{\text {a }}$ as the energy increases. According to [65] this is due to three reasons: First, the number of X-rays per unit $\mathrm{K}_{\mathrm{a} \text { a per unit area }}\left(\Phi / \mathrm{K}_{\mathrm{a}}\right)$ increases as the X-ray energy 
increases up to around $60 \mathrm{keV}$ [6]. Therefore more X-rays (primary signal carriers) are impinging on the scintillator for a given exposure. In our case, it was found that the $\Phi / \mathrm{K}_{\mathrm{a}}$ increases from $4085 \mathrm{X}$-rays $/ \mathrm{mm}^{2} / \mu \mathrm{Gy}$ at $17 \mathrm{keV}$ to $16291 \mathrm{X}$-rays $/ \mathrm{mm}^{2} / \mu \mathrm{Gy}$ at $33 \mathrm{keV}$ (74.9\% increase). Secondly, more secondary quanta (i.e., optical photons) are generated for a higher energy absorbed X-ray photon assuming an almost fixed scintillator's light yield (i.e., optical photons per absorbed energy). In practice, there is a small scintillator light yield nonproportionality, i.e., the photon response of the scintillator changes as a function of energy. In our case, this happens mainly because the energy deposited by the secondary generated electrons changes abruptly in the energies around the K- and L-absorption edges of iodine. It was found that the photon non-proportionality response of the CsI: Tl scintillator changes from around 1.145 at $17 \mathrm{keV}$ to around 1.115 at $33 \mathrm{keV}$ (these values are normalized to unity at 662 $\mathrm{keV)}$ [67], [68], [69]. Combining the non-proportionality values with the light yield of the scintillator, it was calculated that around 1071 and 2024 optical photons are created per absorbed X-ray at photon energies of $17 \mathrm{keV}$ and $33 \mathrm{keV}$, respectively, resulting in $89 \%$ increase. Finally, there is a depth effect, i.e., as the energy increases, the beam becomes more penetrating, so the interacting X-rays are absorbed at deeper points within the scintillator, closer to the digital sensor. Hence, the created optical photons are less likely to be reabsorbed in the scintillator, leading to increased collection efficiency from the digital sensor. For example, assuming that the scintillator consists of 10 layers, only $1.55 \%$ of the X-ray photons are absorbed in the 10th layer at $17 \mathrm{keV}$. The corresponding value for $33 \mathrm{keV}$ is $3.23 \%$, which corresponds to $108.1 \%$ increase. It should be noted that the change in the detector signal per unit $\mathrm{K}_{\text {a }}$ as the energy increases is the combination of the above three reasons and the absorption of X-rays from the scintillator. This is obvious in Fig. 1(b) which shows the gradients of the above STP curves as a function of energy. An almost linear increase up to $33 \mathrm{keV}$ is observed. However, for energies higher than the K-absorption edge of iodine (33.2 keV), we observe much higher slopes due to the increased absorption of X-rays from the scintillator. In particular, the STP slope increases by $53.8 \%$ (from 33 to $33.3 \mathrm{keV}$ ) while the respective DQE(0) value [which takes into account the absorption of X-rays (QDE) and the K-fluorescence X-rays escape and re-absorption (Swank Factor)] increases by 50.8\%, indicating that these two parameters are directly related at energies around the K-absorption edge of iodine.

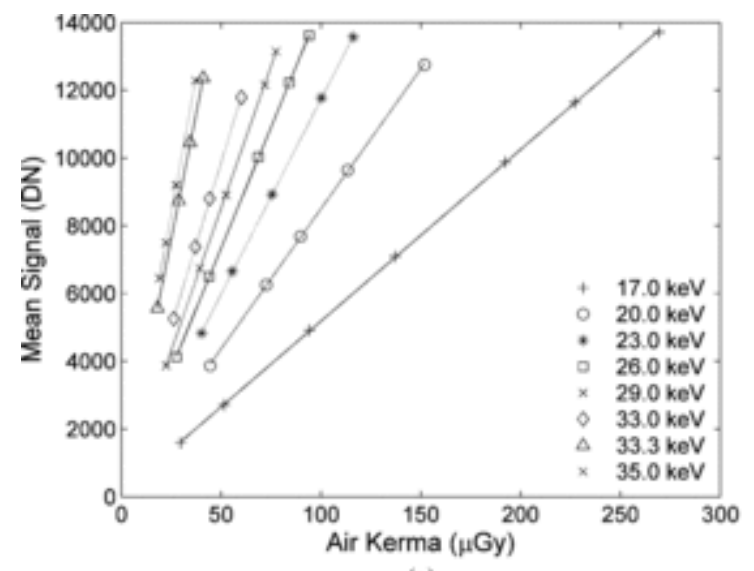

(a)

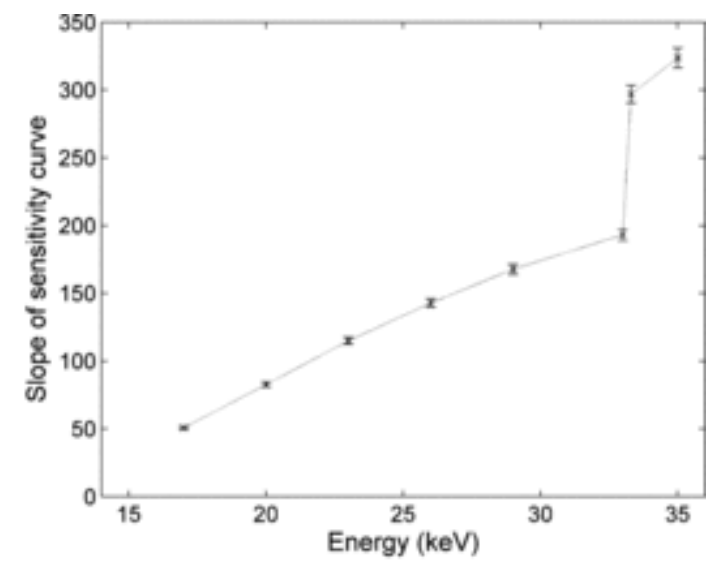

(b)

Fig. 1. (a) STP curves and (b) their slopes as a function of energy. 


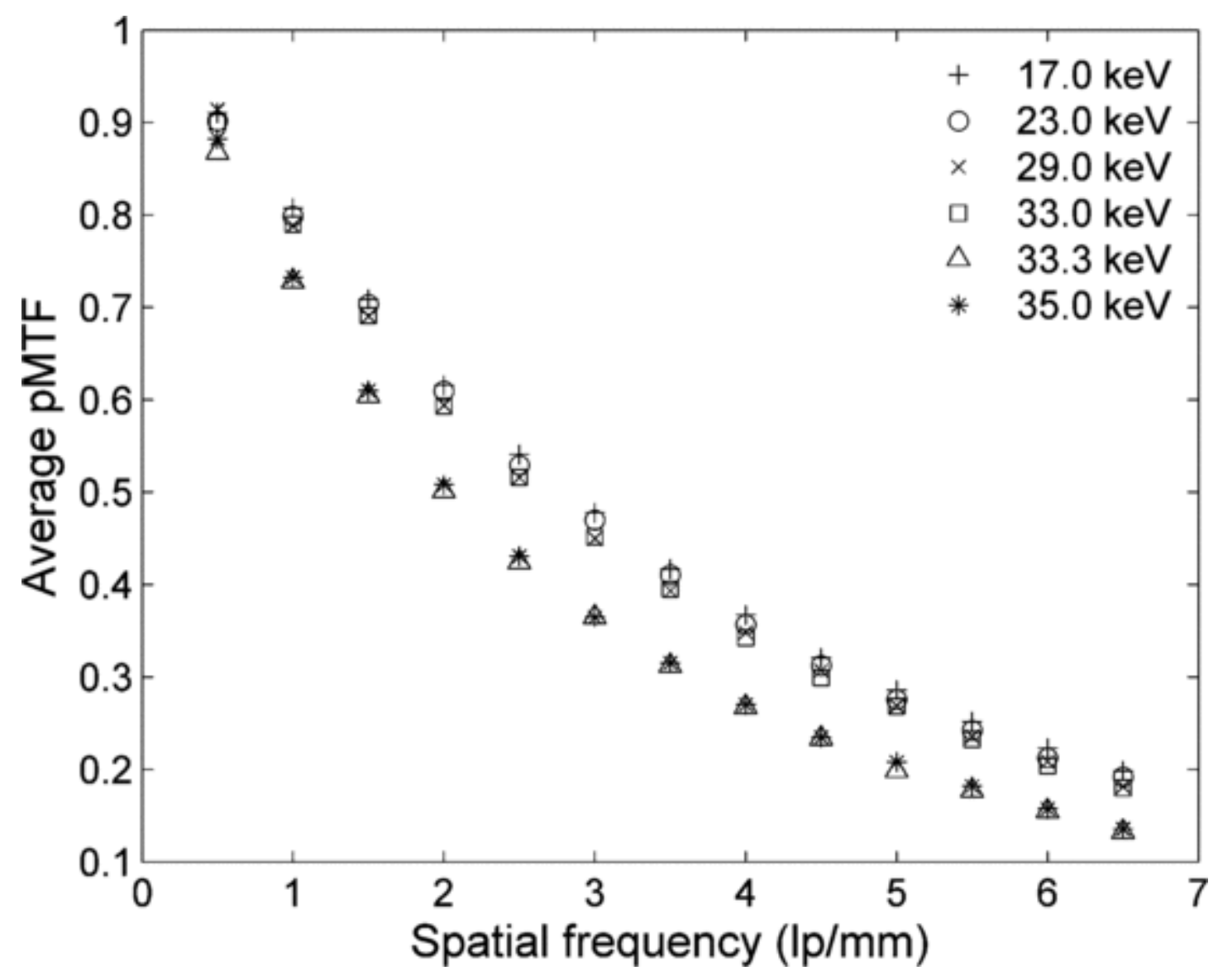

Fig. 2. Average pMTF curves as a function of energy.

\section{Spatial Resolution as a Function of Energy}

Fig. 2 shows the average pMTF curves of the detector over the range of used energies. For energies up to $33.2 \mathrm{keV}$ the pMTF decreases slightly with increasing energy. In particular, the average relative difference between the pMTFs of 17 and $33 \mathrm{keV}$ is less than $5.5 \%$. The most likely explanation for this is the longer range of the photoelectron in the scintillator material. As the columnar structure of the CsI: Tl scintillator prevents the lateral spread of the light photons, the increased X-ray absorption depth as a function of energy does not increase the pMTF, as is the case in granular scintillators such as gadolinium oxysulfide $\left(\mathrm{Gd}_{2} \mathrm{O}_{2} \mathrm{~S}: \mathrm{Tb}\right)$. In the investigated energy range, the pMTF curves reach $50 \%$ at about $3 \mathrm{lp} / \mathrm{mm}$. At energies above $33.2 \mathrm{keV}$, the pMTF values are smaller due to the lateral spread of the K-fluorescence X-ray photons. In this case, the pMTFs reach $50 \%$ at around $2 \mathrm{lp} / \mathrm{mm}$. A comparison between the pMTF curves at 33 and $33.3 \mathrm{keV}$ shows decreasing resolution as a function of spatial frequency from $4 \%$ for $0.5 \mathrm{lp} / \mathrm{mm}$ to $26 \%$ for $6.5 \mathrm{lp} / \mathrm{mm}$. The average relative difference between the pMTFs of 33 and $33.3 \mathrm{keV}$ is $18.5 \%$. A similar behavior was observed in our previous study [무] ], which compared the pMTF curves of CsI: Tl for three X-ray beams: $32 \mathrm{kV}$ using a molybdenum (Mo) anode X-ray tube and $32 \mathrm{keV}$ and $34 \mathrm{keV}$ using monochromatic synchrotron radiation. The pMTF at $35 \mathrm{keV}$ is slightly higher than $33.2 \mathrm{keV}$ (1.5\% average relative difference), probably due to a smaller effect of the K-fluorescence X-ray photons. Finally, it is worth mentioning that the FOP did not introduce a loss of spatial resolution due to the way it is constructed. In particular, a typical FOP consists of multiple single fibers of several $\mu \mathrm{m}$ diameter. Each single fiber consists of a core glass which transports light by reflecting the optical photons. A cladding glass surrounds the core glass and it is enhanced with an extra-mural absorber that absorbs light leakage from the core glass. Hence, the optical photons are prevented from reaching adjacent fibers. 


\section{Noise Assessment}

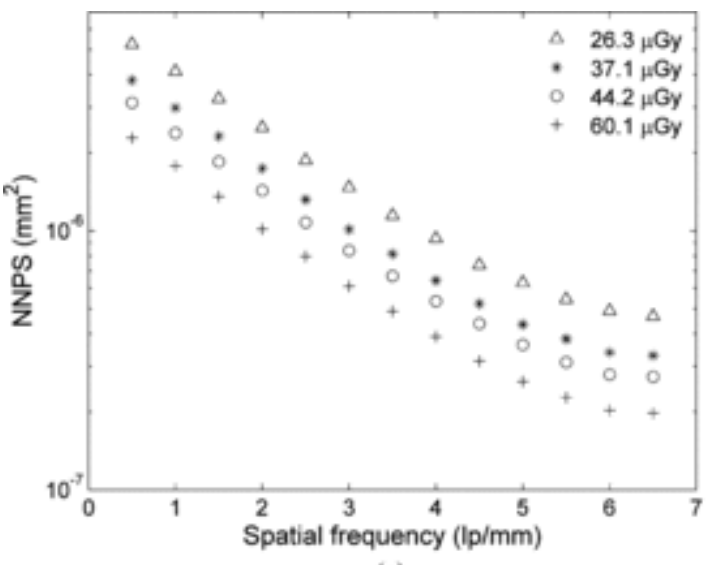

(a)

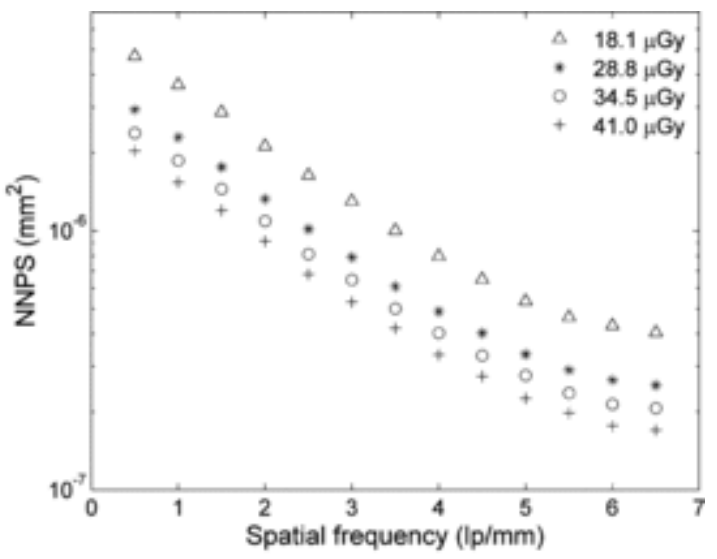

(b)

Fig. 3. Representative average NNPS at (a) $33 \mathrm{keV}$ and (b) $33.3 \mathrm{keV}$.

For brevity's sake, only the NNPS curves at $33 \mathrm{keV}$ and $33.3 \mathrm{keV}$ for HFW mode are shown infig. 3. It can be observed that the NNPS values decrease as a function of $\mathrm{K}_{\text {a }}$ due to the larger increase in the signal compared to the increase in noise. This results in increasing DQE values as a function of exposure, up to a maximum detectability point where the detector exhibits quantum limited behavior (i.e., the X-ray quantum noise is the dominant source of noise). The comparison between the two energies shows that the spectral shape of the NNPS remains the same, i.e., does not seem to be affected by the K-fluorescence X-rays of iodine. However, for

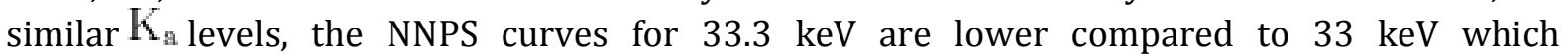
corresponds to higher SNR and DQE values. This happens due to the increased X-ray detectability from the scintillator.

\section{E. X-ray Detectability as a Function of Energy}

Fig. 4 shows the average DQE curves of the detector operated in HFW mode for the energies 17, 20,23 and $26 \mathrm{keV}$. It was found that the uncertainties on the DQE values are less than $8 \%$. However, these have not been included in the figure to avoid cluttering it. It can be observed that the DQE values decrease as a function of energy mainly due to the decreased X-ray detectability from the scintillator. In particular, the DQE $(0.5)$ values are in the range $0.79-0.85$ at $17 \mathrm{keV}, 0.75-0.79$ at $20 \mathrm{keV}, 0.64-0.69$ at $23 \mathrm{keV}$, and $0.55-0.60$ at $26 \mathrm{keV}$. Other reasons leading to decreased DQE values as a function of energy are 1) the decrease in the PMTF, 2) a change in the NPS, and 3) an increase in the $\Phi / \mathrm{K}_{\mathrm{a}}[\underline{71}]$. Fig. 5 shows the average DQE curves of the detector (HFW mode) for the energies 29, 33, 33.3 and $35 \mathrm{keV}$. In this case, the uncertainties on the DQE results are less than 10\%. For the first two energies the DQE values decrease as a function of energy due to the decreased detectability of X-rays from the scintillator. The $\mathrm{DQE}(0.5)$ values are in the range $0.46-0.50$ at $29 \mathrm{keV}$ and $0.35-0.36$ at $33 \mathrm{keV}$. At higher energies, the DQE values are increased due to the K-absorption edge of iodine. More specifically, the DQE(0.5) values are in the range $0.54-0.57$ at $33.3 \mathrm{keV}$ and $0.53-0.55$ at $35 \mathrm{keV}$. We can observe that the higher energy DQE curves have different spectral shape compared to the lower ones. This happens due to the spread of the K-fluorescence photons that decrease the spatial resolution of the detector (see Fig. 2).

Fig. 6 compares the maximum DQE values (i.e., at $0.5 \mathrm{lp} / \mathrm{mm}$ ) in HFW operation mode to the analytically calculated QDE/EAE and the Monte Carlo calculated DQE(0) of the scintillator. The maximum $\mathrm{DQE}(0)$ has not been extracted from the experimental data because it is difficult to 
completely remove the excessive large area signal at zero spatial frequency (seeSection IIE). Previous studies estimated the $\mathrm{DQE}(0)$ values by applying either first-order linear extrapolation [72] or second-order polynomial extrapolation [65] to the measured DQE curves. However, in the current work it was found that the extrapolation does not have the same effect on all curves,
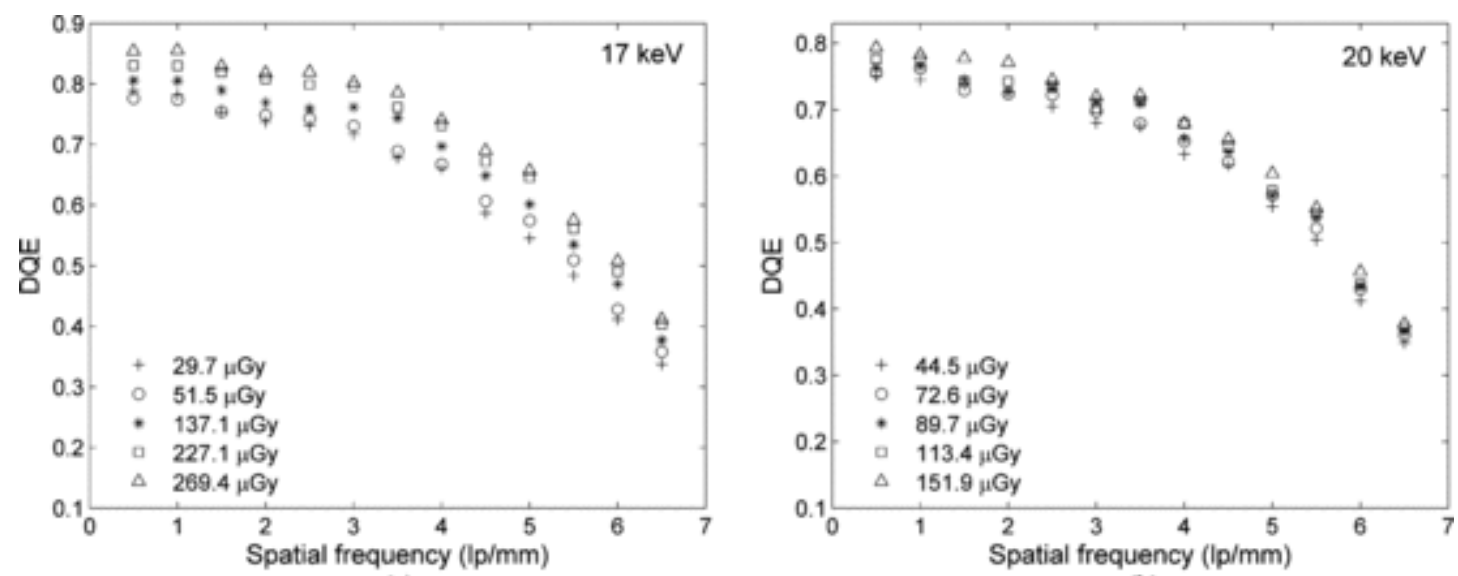

(a)

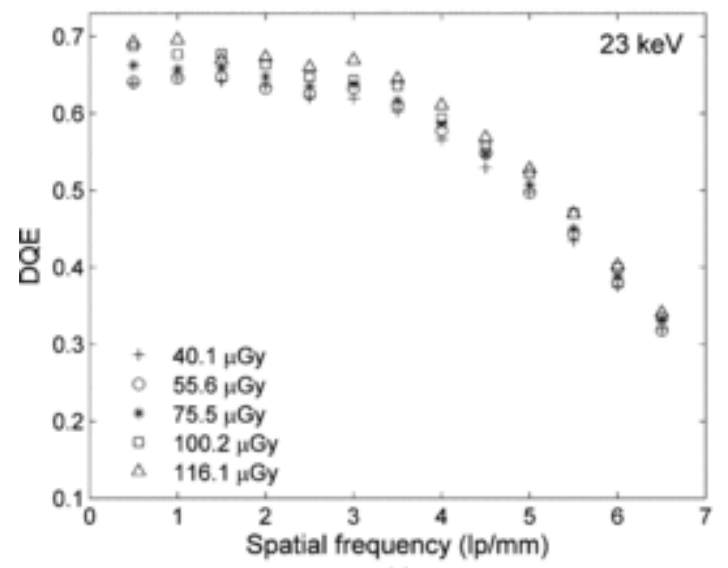

(b)

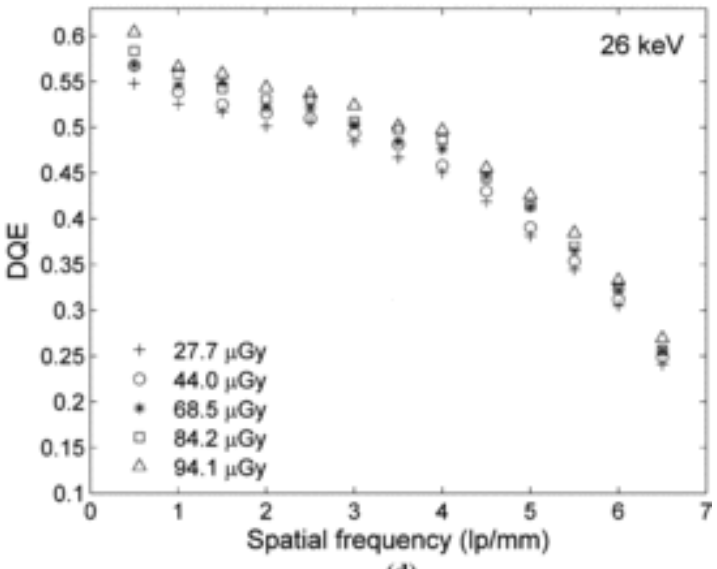

(d)

Fig. 4. Average DQE at (a) $17 \mathrm{keV}$, (b) $20 \mathrm{keV}$, (c) $23 \mathrm{keV}$, and (d) $26 \mathrm{keV}$ (HFW mode). 

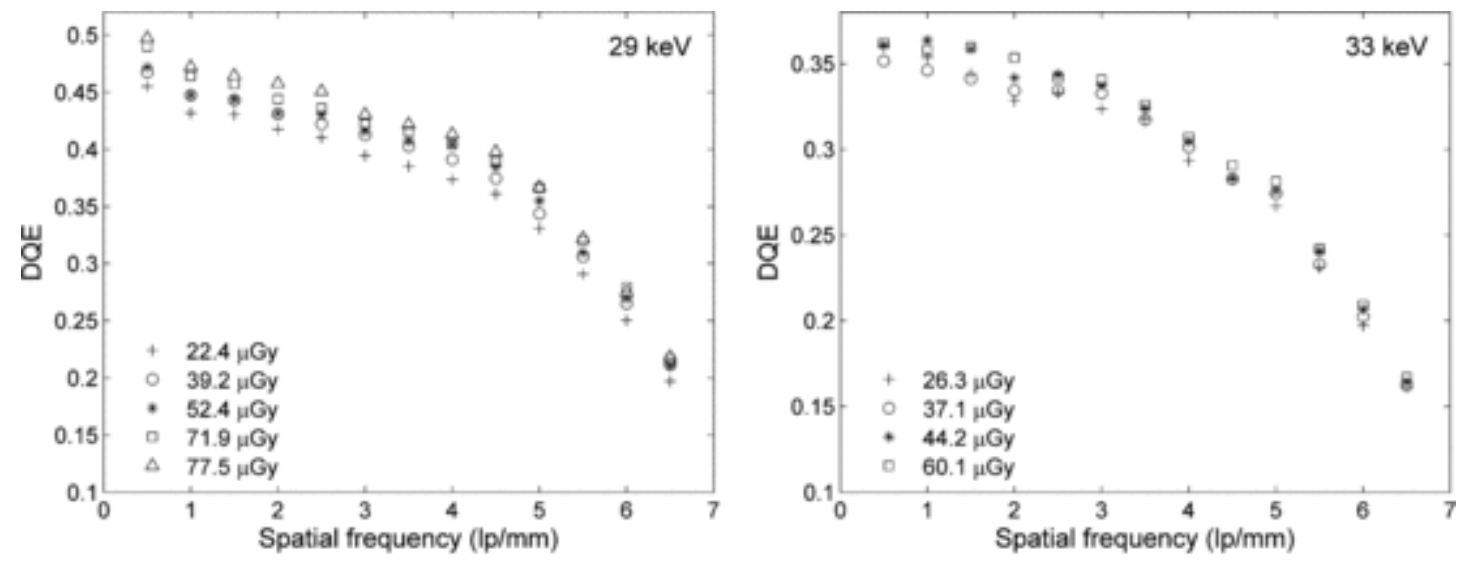

(a)

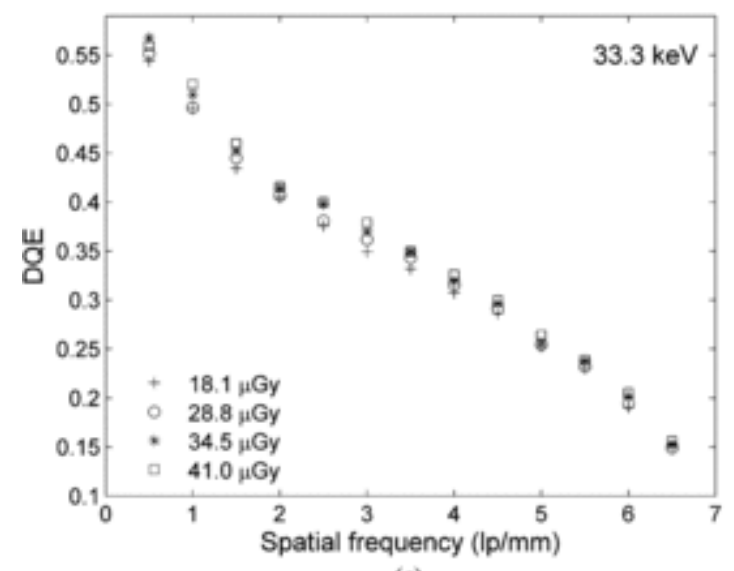

(b)

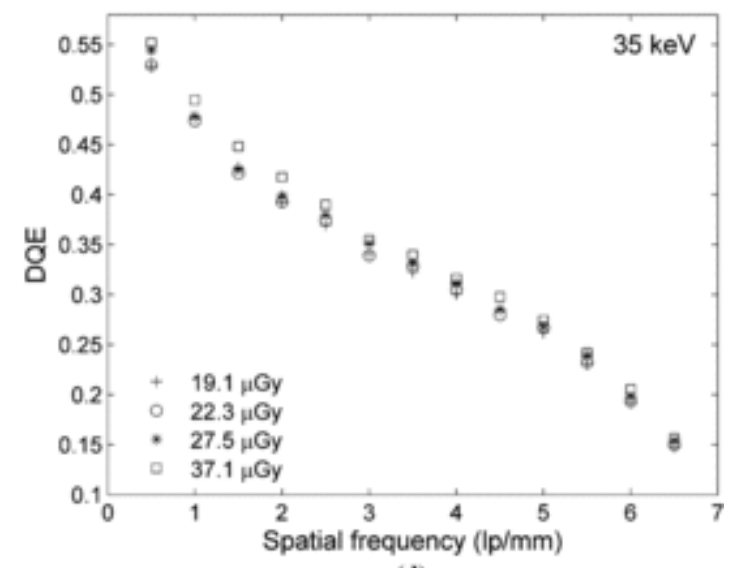

(c)

(d)

Fig. 5. Average DQE at (a) $29 \mathrm{keV}$, (b) $33 \mathrm{keV}$, (c) $33.3 \mathrm{keV}$, and (d) $35 \mathrm{keV}$ (HFW mode).

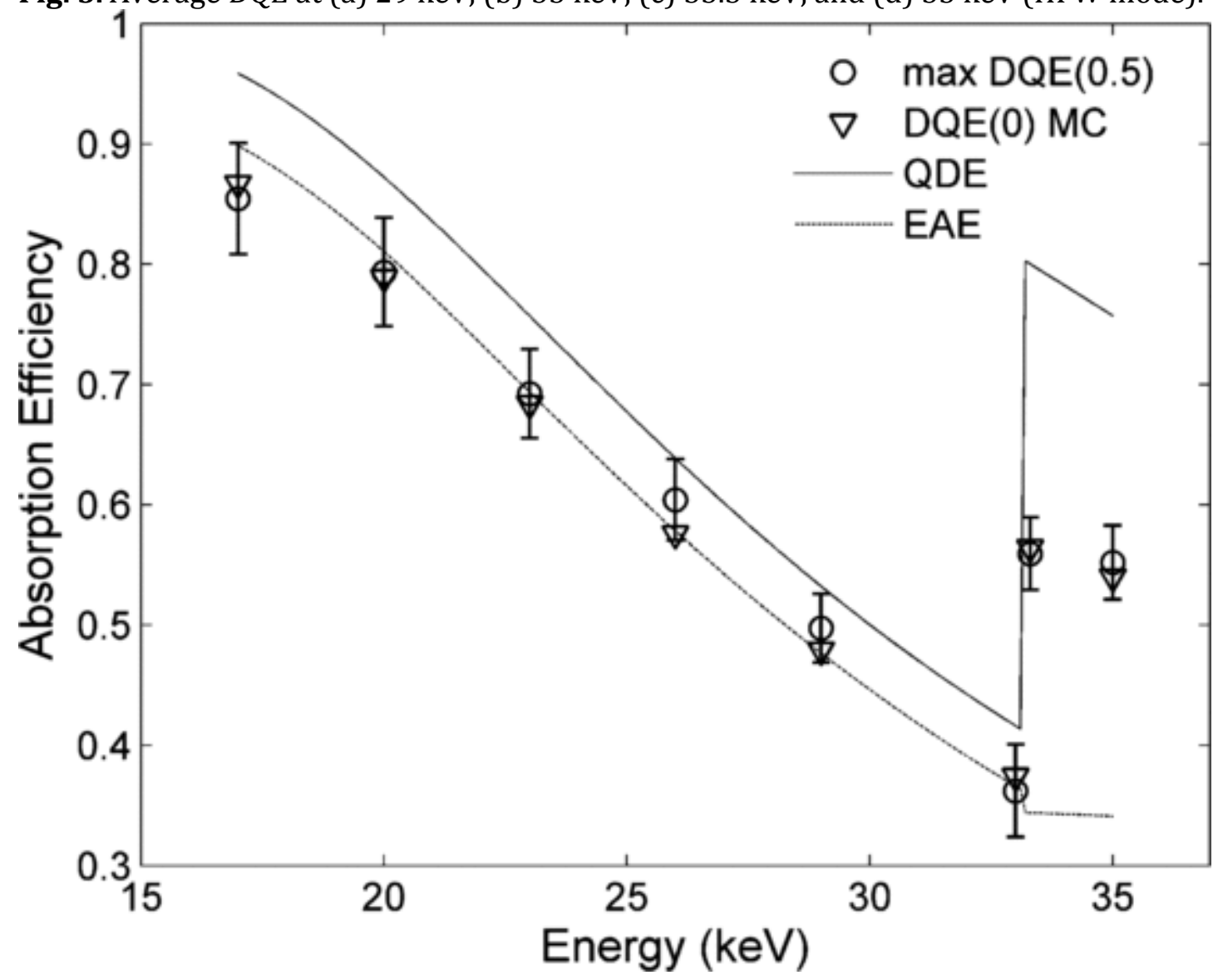


Fig. 6. Maximum DQE(0.5) compared to the analytical QDE/EAE and DQE(0) MC.

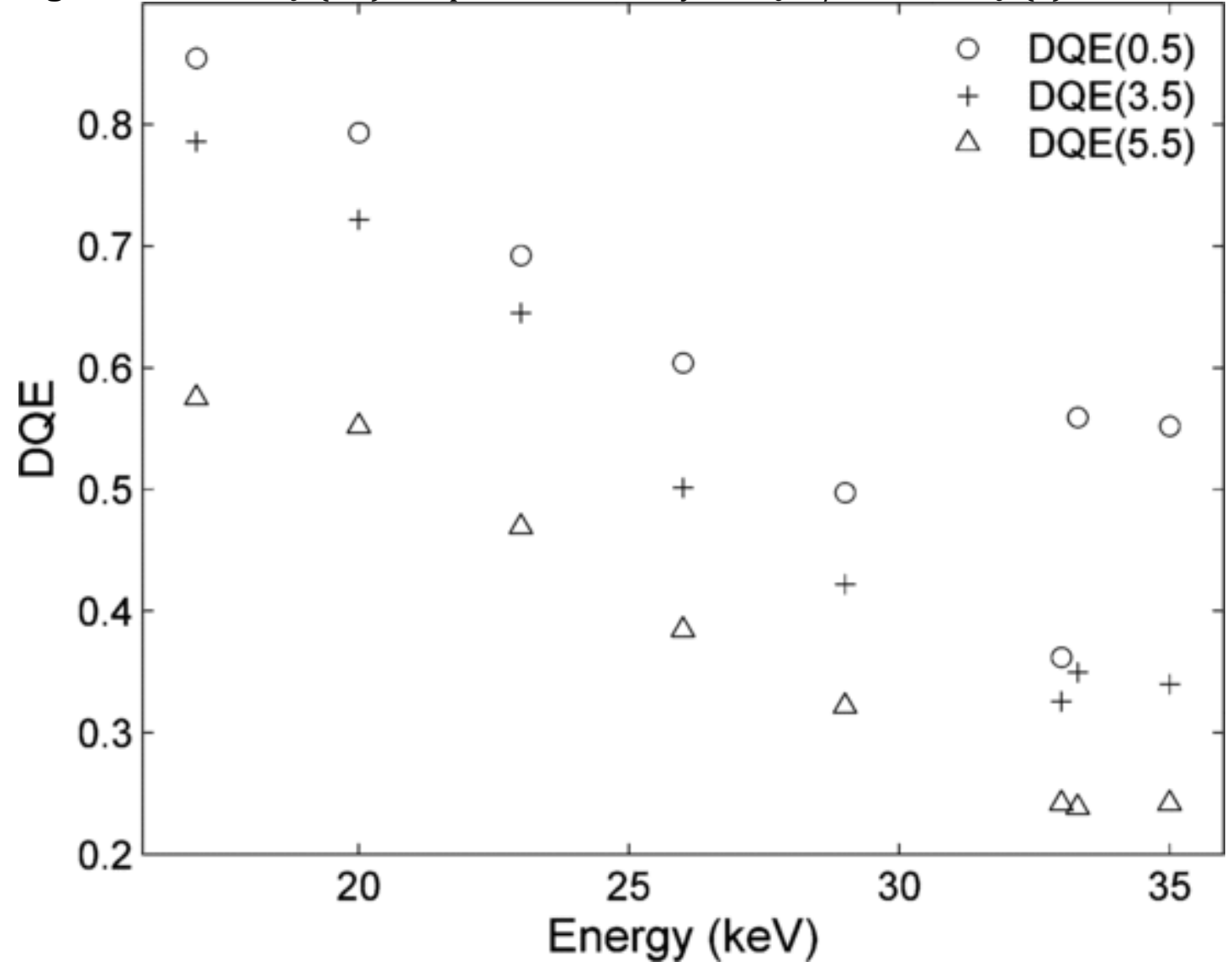

Fig. 7. DQE as a function of energy for three spatial frequencies.

\begin{tabular}{|c|c|c|c|c|c|c|c|c|c|c|}
\hline $\begin{array}{l}\text { Energy } \\
\text { (keV) }\end{array}$ & $\max _{\text {DQE(0.5) }}^{\max }$ & QDE & $\begin{array}{c}\text { (\%) QDE vs } \\
\text { DQE }(0.5)\end{array}$ & EAE & $\begin{array}{l}(\%) \text { E.AE VS } \\
\text { DQE }(0.5)\end{array}$ & QDE MC & $I_{A F D}$ & $\mathbf{I}_{\text {OPD }}$ & DQE(0) MC & $\begin{array}{c}\text { (\%) DQE(0) } \\
\text { MC vs } \\
\text { DQE(0.5) }\end{array}$ \\
\hline 17 & 0.855 & 0.959 & -12.172 & 0.899 & -5.153 & 0.959 & 0.999 & 0.905 & 0.867 & -1.449 \\
\hline 20 & 0.794 & 0.872 & -9.946 & 0.811 & -2.168 & 0.872 & 0.999 & 0.905 & 0.789 & 0.572 \\
\hline 23 & 0.692 & 0.757 & -9.358 & 0.694 & -0.176 & 0.756 & 0.999 & 0.905 & 0.684 & 1.208 \\
\hline 26 & 0.604 & 0.639 & -5.734 & 0.578 & 4.298 & 0.637 & 0.998 & 0.905 & 0.576 & 4.653 \\
\hline 29 & 0.497 & 0.532 & -6.992 & 0.476 & 4.196 & 0.532 & 0.996 & 0.905 & 0.479 & 3.691 \\
\hline 33 & 0.362 & 0.416 & -14.937 & 0.366 & -1.102 & 0.416 & 0.994 & 0.905 & 0.374 & -3.280 \\
\hline 33.3 & 0.559 & 0.800 & -43.063 & 0.344 & 38.553 & 0.803 & 0.777 & 0.905 & 0.564 & -0.824 \\
\hline 35 & 0.552 & 0.757 & -37.189 & 0.341 & 38.239 & 0.757 & 0.788 & 0.905 & 0.54 & 2.181 \\
\hline
\end{tabular}

TABLE I COMPARISON BETWEEN

THE MAXIMUM DQE(0.5) VALUES

AND THE QDE/EAE AND DQE(0) MC 


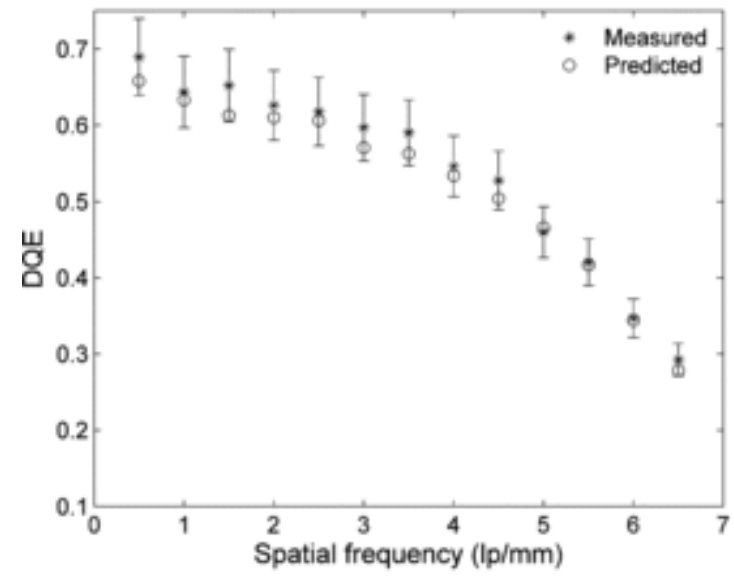

(a)

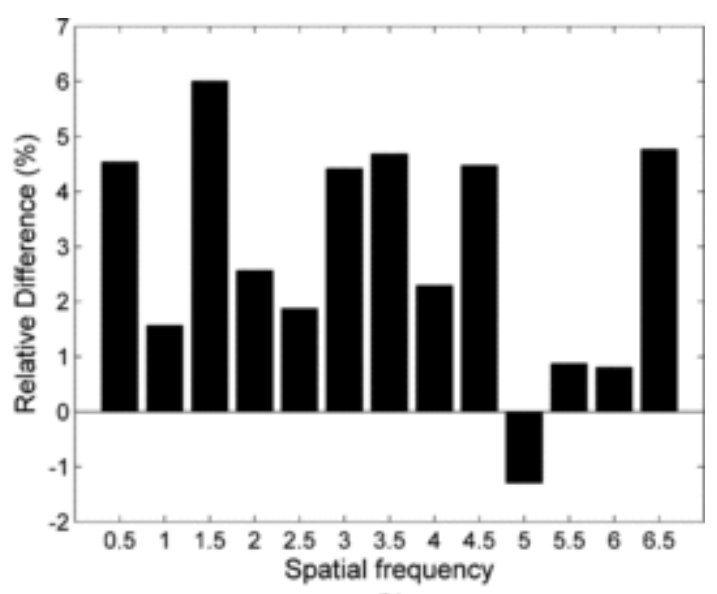

(b)

Fig. 8. (a) Predicted compared to measured DQE values at $28 \mathrm{kV}$ W/Al (HFW mode, $111 \mu \mathrm{Gy}$ ) and (b) their relative difference (\%).

\begin{tabular}{lcccc}
\hline Detector name & $\begin{array}{c}\text { Radiation } \\
\text { quality }\end{array}$ & $\begin{array}{c}\mathbf{p M T F}(\mathbf{0 . 5}) \\
(\mathbf{l p} / \mathbf{m m})\end{array}$ & DQE peak & $\begin{array}{c}\mathbf{K}_{\mathbf{a}} \text { at detector } \\
(\boldsymbol{\mu G \mathbf { G } )}\end{array}$ \\
\hline $\begin{array}{c}\text { GE Senographe } \\
\text { 2000D }\end{array}$ & $28 \mathrm{kV} \mathrm{Rh/Rh}$ & 3.2 & 0.41 & 91 \\
$\begin{array}{c}\text { GE Senographe } \\
\text { DS }\end{array}$ & $28 \mathrm{kV} \mathrm{Mo} / \mathrm{Mo}$ & 3.3 & 0.40 & 86 \\
$\begin{array}{c}\text { GE Essential } \\
\text { Dexela } 2923\end{array}$ & $29 \mathrm{kV} \mathrm{Rh} / \mathrm{Rh}$ & 2.4 & 0.59 & 92 \\
Dexela 2923 & $28 \mathrm{kV} \mathrm{W} / \mathrm{Al}$ & 3.3 & 0.69 & 89 \\
Dexela 2923 & $23 \mathrm{keV}$ & 2.8 & 0.69 & 100 \\
\hline
\end{tabular}

TABLE II COMPARISON OF THE DEXELA DETECTOR WITH THREE COMMERCIALLY AVAILABLE FPDS

leading to unsatisfactory extrapolations at $0 \mathrm{lp} / \mathrm{mm}$. It was found that the $\%$ difference between DQE(0) and DQE(0.5) was in the range 1-6\%. Therefore, it was decided to use DQE(0.5) as the minimum spatial frequency DQE. It can be observed that the EAE describes well the maximum detectability of the system for energies below the K-absorption edge of the iodine. However, at higher energies, it severely underestimates the detectability (seeSection II-C). On the other hand, the QDE overestimates the maximum detectability especially at energies higher than 33.2 $\mathrm{keV}$. Conversely, the Monte Carlo (MC) calculated DQE(0) estimates accurately the maximum detectability of the system for all energies.

Table I further quantifies the above by comparing the analytical QDE/EAE and DQE $(0)$ MC to the experimentally measured $\mathrm{DQE}(0.5)$. It can be observed that the $\mathrm{DQE}(0.5)$ is $43 \%$ lower 
compared to the QDE at $33.3 \mathrm{keV}$, while it is $39 \%$ higher than the EAE at the same energy. On the other hand, the maximum difference between the measured DQE(0.5) and DQE(0) MC is less than $5 \%$, which is within the accepted precision on the DQE calculation (10\%) from the IEC standard [57]. These results demonstrate that at very small spatial frequencies, where the spatial resolution does not have any effect, the fraction of input X-ray quanta used to create the output image is limited only by the X-ray detectability of the front-end (scintillator) rather than the noise of the back-end (CMOS sensor) part of the X-ray detector.

Fig. 7 shows how the DQE values at three spatial frequencies $(0.5,3.5$ and $5.5 \mathrm{lp} / \mathrm{mm})$ change as

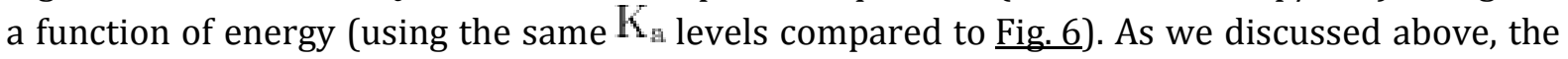
$\mathrm{DQE}(0.5)$ is affected only by the X-ray absorption from the scintillator. However, the DQE at higher spatial frequencies is also affected by the ratio of pMTF to NNPS, i.e., the combination of contrast, spatial resolution and noise. The DQE decreases as a function of spatial frequency mainly due to the increasing effect of noise [궁. At high spatial frequencies (e.g., $5.5 \mathrm{lp} / \mathrm{mm}$ ) the $\mathrm{K}$-absorption edge effect on the DQE values is not significant.

It is worth mentioning that this type of analysis also offers the possibility to predict the X-ray performance of the detector for any mammographic beam quality within the investigated energy range. As an example, we used interpolation, extrapolation and the normalized spectral shape that corresponds to $\mathrm{W} / \mathrm{Al}$ at $28 \mathrm{kV}$ (as a weighting factor) to predict the horizontal DQE of the detector (in HFW mode) at $111 \mu \mathrm{Gy}$ [Fig. 8(a)]. The maximum relative difference between the predicted and the measured (taken from [이]) horizontal DQE values was 6\% [Fig. 8(b)]. This demonstrates that the data acquired at monochromatic energies could be combined to calculate the pMTF, NNPS, and DQE at different mammographic spectral shapes (i.e., different radiographic beam qualities). In turn, these parameters can be given as input to an image simulation program [40], [4] to predict the effect of the X-ray beam on image quality (e.g., in terms of contrast-to-noise ratio, contrast-detail analysis, detectability index, etc.). Table II compares the X-ray performance (in terms of spatial frequency corresponding to $50 \%$ MTF and DQE peak) of the Dexela detector with that of three commercially available hydrogenated amorphous silicon (a-Si:H) based FPDs [75]. All three FPD detectors have pixel pitches equal to $100 \mu \mathrm{m}$ and are optically coupled to CsI scintillators. Their X-ray performances were measured using either Mo/Mo at $28 \mathrm{kV}$ or rhodium/rhodium (Rh/Rh) at 28 and $29 \mathrm{kV}$. Hence, the average energies of the used spectra were around $20 \mathrm{keV}$ due to the combination of the K-fluorescence X-rays of Mo ( $\mathrm{K}_{\alpha}$ at $17.4 \mathrm{keV}$ and $\mathrm{K}_{\beta}$ at $\left.19.6 \mathrm{keV}\right)$ and $\mathrm{Rh}\left(\mathrm{K}_{\alpha}\right.$ at $20.2 \mathrm{keV}$ and $\mathrm{K}_{\beta}$ at $\left.22.7 \mathrm{keV}\right)$ with the K-absorption edges of Mo $(20.0 \mathrm{keV})$ and $\mathrm{Rh}(23.3 \mathrm{keV})$, respectively. Therefore, their performances can be compared with that of the Dexela detector at $20 \mathrm{keV}$. In the same table we also compare the monochromatic (23 keV) with the polychromatic $(\mathrm{W} / \mathrm{Al}$ at $28 \mathrm{kV}$, average energy equal to $22.8 \mathrm{keV}$-taken from [무]]) X-ray performance of the Dexela detector. It can be observed that the spatial resolution of the Dexela detector at $20 \mathrm{keV}$ (where it reaches $50 \% \mathrm{pMTF}$ at $2.8 \mathrm{lp} / \mathrm{mm}$ ) is comparable to that of commercially available FPDs (which reach $50 \%$ pMTF at $2.5-3.3 \mathrm{lp} / \mathrm{mm}$ ). On the other hand, the Dexela detector demonstrates a higher DQE peak value (0.76) compared with the FPDs (0.41-0.59). Finally, the investigated detector at $23 \mathrm{keV}$ demonstrates similar PMTF and DQE values compared with the polychromatic ones [39]. This is another demonstration that the DQE values of any mammographic spectrum can be predicted from the monochromatic DQE data. It is worth mentioning that the DQE peak of the Dexela detector at $23 \mathrm{keV}$ is higher compared to those of the FPDs, despite the reduced X-ray absorption from the scintillator.

\section{CONCLUSIONS}

The purpose of this study was to evaluate the performance of a CMOS APS X-ray detector in a range of monochromatic energies (17-35 keV), and give insight on phenomena that cannot be 
observed directly with a polychromatic spectrum, i.e., how the pMTF, NNPS, and DQE values change as a function of energy, in particular in the vicinity of the iodine K-edge. For this reason, a synchrotron light source was used to provide eight distinct energies below and above the Kabsorption edge of iodine. It was found that the maximum low frequency DQE values of the detector (operated in HFW mode) are similar to the calculated X-ray detectability of the scintillator, which indicates quantum limited behavior of the sensor. This means that the electronic noise of the CMOS sensor is marginal and the measurement floor is only due to the scintillator (front-end) rather than the CMOS sensor (back-end of the X-ray detector). However, at higher spatial frequencies, the DQE also depends on the relationship between the pMTF and NNPS, as illustrated in the current study. The high DQE performance, compared to commercially available a-Si:H FPDs, indicates the suitability of the detector in many low energy applications. The increased X-ray detectability of the system due to the $\mathrm{K}$-absorption edge of iodine is promising for contrast enhanced mammographic applications such as dual energy and digital subtraction mammography (DSM). It should be noted that the pMTF values decrease at energies above $33.2 \mathrm{keV}$. However, the decreased resolution is not the most important parameter in dual energy or temporal subtraction mammography, which aim to depict vessels down to $3 \mathrm{~mm}$ diameter [76], [77]. Mammographic and breast tomosynthesis phantom images captured with the same detector and monochromatic synchrotron radiation will be the subject of a future publication. Finally, we demonstrated that the acquired data at monochromatic energies enable the calculation of pMTF, NNPS, and DQE at different spectral shapes within the investigated energy range. In turn, these can be used to predict the effect of the X-ray spectral shape on mammographic image quality by means of image simulation based on the experimentally measured pMTF, NNPS, and SNR.

\section{AUTHORS/ ACKNOLWEDGEMENTS}

The work of M. B. Szafraniec was supported in part by an EPSRC-GB doctoral training allowance. The work of A. Olivo was supported by the UK Engineering and Physical Sciences Research Council grants (EP/G004250/1 and EP/1021884/1). The detector was supported by Dexela Limited (a PerkinElmer company).

A. C. Konstantinidis, M. B. Szafraniec, R. D. Speller, and A. Olivo are with the Department of Medical Physics and Bioengineering, University College London, London WC1E 6BT, U.K. (email:anastasios.konstantinidis@ucl.ac.uk).

L. Rigon is with the Istituto Nazionale di Fisica Nucleare, Sezione di Trieste, Trieste 34127, Italy (e-mail: luigi.rigon@ts.infn.it).

G. Tromba, D. Dreossi, and N. Sodini are with the ELETTRA, Trieste 34012, Italy (email:giuliana.tromba@elettra.trieste.it).

P. F. Liaparinos is with the Department of Medical Instruments Technology, Technological Educational Institution of Athens, Athens 122 10, Greece (e-mail: liapkin@teiath.gr).

S. Naday, S. Gunn, and A. McArthur are with Dexela Limited (a PerkinElmer company), London N1 7EU, U.K. 


\section{REFERENCES}

1. T. Graeve and G. P. Weckler

"High-resolution CMOS imaging detector"

Proc. SPIE, vol. 4320, pp. 68-76, 2001

2. H. K. Kim, G. Cho , S. W. Lee, Y. H. Shin and H. S. Cho

"Development and evaluation of a digital radiographic system based on CMOS image sensor"

IEEE Trans. Electron Devices, vol. 48, pp. 662-666, 2001

3. S. Mendis , S. E. Kemeny and E. R. Fossum

"CMOS active pixel image sensor"

IEEE Trans. Electron Devices, vol. 41, pp. 452-453, 1994

4. D. W. Holdsworth , R. K. Gerson and A. Fenster

"A time-delay integration charge-coupled device camera for slot-scanned digital radiography"

Med. Phys., vol. 17, pp. 876-886, 1990

5. L. E. Antonuk

"Demonstration of megavoltage and diagnostic x-ray imaging with hydrogenated amorphous silicon arrays"

Med. Phys., vol. 19, pp. 1455-1466, 1992

6. M. J. Yaffe and J. A. Rowlands

"X-ray detectors for digital radiography"

Phys. Med. Biol., vol. 42, pp. 1-39, 1997

7. M. Spahn

"Flat detectors and their clinical applications"

Eur. Radiol., vol. 15, pp. 1934-1947, 2005

8. M. Bigas , E. Cabruja , J. Forest and J. Salvi

"Review of CMOS image sensors"

J. Microelect., vol. 37, pp. 433-451, 2006

9. A. El Gamal and H. Eltoukhy

"CMOS image sensors"

IEEE Circuits Devices, vol. 21, pp. 6-20, 2005

10. M. B. Williams

"Analysis of the detective quantum efficiency of a developmental detector for digital mammography"

Med. Phys., vol. 26, pp. 2273-2285, 1999

11. J. R. Janesick

"Lux transfer: Complementary metal oxide semiconductors versus charge-coupled devices"

Opt. Eng., vol. 41, pp. 1203-1215, 2002

12. R. A. Close , K. C. Shah and J. S. Whiting

"Regularization method for scatter-glare correction in fluoroscopic images"

Med. Phys., vol. 26, pp. 1794-1801, 1999 
13. R. R. Liu , S. Rudin and D. R. Bednarek

"Super-global distortion correction for a rotational C-arm x-ray image intensifier"

Med. Phys., vol. 26, pp. 1802-1810, 1999

14. J. R. Janesick

Scientific Charge-Coupled Devices

pp. 3-93, 2001, SPIE

15. W. Zhao and J. A. Rowlands

"Large-area solid state detector for radiology using amorphous selenium"

Proc. SPIE, vol. 1651, pp. 134-143, 1992

16. L. E. Antonuk

"Strategies to improve the signal and noise performance of active matrix, flat-panel imagers for diagnostic x-ray applications"

Med. Phys., vol. 27, pp. 289-306, 2000

17. F. Busse

"Design and performance of a high-quality cardiac flat detector"

Proc. SPIE, vol. 4682, pp. 819-827, 2002

18. D. Scheffer

"A wafer scale active pixel CMOS image sensor for generic X-ray radiology"

Proc. SPIE, vol. 6510, pp. U233-U241, 2007

19. J. H. Siewerdsen and D. A. Jaffray

"A ghost story: Spatio-temporal response characteristics of an indirect-detection flat-panel imager"

Med. Phys., vol. 26, pp. 1624-1641, 1999

20. A. K. Bloomquist, M. J. Yaffe , G. E. Mawdsley , D. M. Hunter and D. J. Beideck

"Lag and ghosting in a clinical flat-panel selenium digital mammography system"

Med. Phys., vol. 33, pp. 2998-3005, 2006

21. E. S. Said

"Design and characterization of ionizing radiation tolerant CMOS APS image sensors up to 30 Mrd (Si) total dose: Part 1"

IEEE Trans. Nucl. Sci., vol. 48, pp. 1796-1806, 2001

22. E. R. Fossum

"CMOS image sensors: Electronic camera-on-a-chip"

IEEE Trans. Electron Devices, vol. 44, pp. 1689-1698, 1997

23. J. R. Janesick

"Charge coupled CMOS and hybrid detector arrays"

Proc. SPIE, vol. 5167, pp. 1-18, 2003

24. A. D. Falcone , Z. Prieskorn , C. Griffith , S. Bongiorno and D. N. Burrows

"Recent progress on developments and characterization of hybrid CMOS x-ray detectors"

Proc. SPIE, vol. 8453, pp. 84530E-1-84530E-7, 2012

25. Z. Prieskorn , C. V. Griffith , S. D. Bongiorno , A. D. Falcone and D. N. Burrows

"Characterization of Si hybrid CMOS detectors for use in the soft x-ray band"

Nucl. Instrum. Meth. A, 
26. J. G. Mainprize

"A CdZnTe slot-scanned detector for digital mammography"

Med. Phys., vol. 29, pp. 2767-2781, 2002

27. J. De Vos

"On the processing aspects of high performance hybrid backside illuminated CMOS imagers"

J. Micromech. Microeng., vol. 21, pp. 1-7, 2011

28. M. Skarpathiotakis

"Development of contrast digital mammography"

Med. Phys., vol. 29, pp. 2419-2426, 2002

29. J. M. Lewin , P. K. Isaacs , V. Vance and F. J. Larke

"Dual-energy contrast-enhanced digital subtraction mammography: Feasibility"

Radiology, vol. 229, pp. 261-268, 2003

30. L. T. Niklason

"Digital tomosynthesis in breast imaging"

Radiology, vol. 205, pp. 399-406, 1997

31. J. T. Dobbins IIIand D. J. Godfrey

"Digital X-ray tomosynthesis: Current state of the art and clinical potential"

Phys. Med. Biol., vol. 48, pp. R65-R106, 2003

32. T. Wu

"Tomographic mammography using a limited number of low-dose cone-beam projection images"

Med. Phys., vol. 30, pp. 365-380, 2003

33. P. F. Liaparinos , I. S. Kandarakis , D. A. Cavouras , H. B. Delis and G. S. Panayiotakis

"Modeling granular phosphor screens by Monte Carlo methods"

Med. Phys., vol. 33, pp. 4502-4514, 2006

34. P. F. Liaparinos and I. S. Kandarakis

"The imaging performance of compact $\$\left\{\backslash \mathrm{rm} \quad \mathrm{Lu}_{-}\{2\}\{\backslash \mathrm{rm} \quad 0\}_{-}\{3\}:\{\backslash \mathrm{rm} \quad\right.$ Eu\} $\$$ powdered phosphor screens: Monte Carlo simulation for applications in mammography"

Med. Phys., vol. 36, pp. 1985-1997, 2009

35. P. F. Liaparinos and I. S. Kandarakis

"Overestimations in zero frequency DQE of X-ray imaging converters assessed by Monte Carlo techniques based on the study of energy impartation events"

Med. Phys., vol. 38, pp. 4440-4450, 2011

36. N. Yagi , M. Yamamoto , K. Uesugi and K. Inoue

"A large-area CMOS imager as an X-ray detector for synchrotron radiation experiments"

J. Synchrotron Rad., vol. 11, pp. 347-352, 2004

37. N. Yagi and K. Inoue

"CMOS flatpanel detectors for SAXS/WAXS experiments"

J. Appl. Cryst., vol. 40, pp. s439-s441, 2007

38. A. C. Thompson, J. C. Nix , T. G. Achterkirchen and E. M. Westbrook

"Large format CMOS-based detectors for diffraction studies"

J. Phys.: Conf. Series, vol. 425, pp. 1-4, 2013 
39. A. C. Konstantinidis , M. B. Szafraniec , R. D. Speller and A. Olivo

"The Dexela 2923 CMOS X-ray detector: A flat panel detector based on CMOS active pixel sensors for medical imaging applications"

Nucl. Instrum. Meth. A, vol. 689, pp. 12-21, 2012

40. A. C. Konstantinidis

Evaluation of digital $\mathrm{x}$-ray detectors for medical imaging applications

2011

41. V. V. Nagarkar , J. S. Gordon, S. Vasile , J. Xie and W. C. Phillips

"Improved X-ray converters for CCD-based crystallography detectors"

Proc. SPIE, vol. 2519, pp. 2-11, 1995

42. A. Badano and J. Sempau

"MANTIS: Combined x-ray, electron and optical Monte Carlo simulations of indirect radiation imaging systems"

Phys. Med. Biol., vol. 51, pp. 1545-1561, 2006

43. V. V. Nagarkar , T. K. Gupta, S. R. Miller, Y. Klugerman , M. R. Squillante and G. Entine

"Structured CsI (Tl) scintillators for X-ray imaging applications"

IEEE Trans. Nucl. Sci., vol. 45, pp. 492-496, 1998

44. J. A. Rowlands and J. Yorkston J. Beutel, H. L. Kundel and R. L. Van Metter

Handbook of Medical Imaging Physics and Psychophysics

vol. 1, pp. 244-248, 2000, SPIE

45. J. M. Boone J. Beutel , H. L. Kundel and R. L. Van Metter

Handbook of Medical Imaging Physics and Psychophysics

vol. 1, pp. 67-69, 2000, SPIE

46. J. H. Hubbel and S. M. Seltzer; Tables of X-ray Mass Attenuation Coefficients and Mass Energy Absorption Coefficients $1 \mathrm{keV}$ to $20 \mathrm{MeV}$ for Elements $\mathrm{Z}=1$ to 92 and 48 Additional Substances of Dosimetric Interest, 1995

47. R. K. Swank

"Absorption and Noise in X-ray Phosphors"

J. Appl. Phys., vol. 44, pp. 4199-4203, 1973

48. H. P. Chan and K. Doi

"Studies of x-ray-energy absorption and quantum noise properties of x-ray screens by use of Monte-Carlo simulation"

Med. Phys., vol. 11, pp. 37-46, 1984

49. W. Zhao, G. Ristic and J. A. Rowlands

"X-ray imaging performance of structured cesium iodide scintillators"

Med. Phys., vol. 31, pp. 2594-2605, 2004

50. A. R. Lubinsky, W. Zhao , G. Ristic and J. A. Rowlands

"Screen optics effects on detective quantum efficiency in digital radiography: Zero-frequency effects"

Med. Phys., vol. 33, pp. 1499-1509, 2006

51. A. Abrami

"Medical applications of synchrotron radiation at the SYRMEP beamline of ELETTRA"

Nucl. Instrum. Meth. A, vol. 548, pp. 221-227, 2004 
52. F. Arfelli

"Mammography with synchrotron radiation: Phase-detection techniques"

Radiology, vol. 215, pp. 286-293, 2000

53. G. Tromba

"The SYRMEP beamline of elettra: Clinical mammography and bio-medical applications"

Proc. 6th Int. Conf. Med. Appl. Synch. Radiation, vol. 1266, pp. 18-23, 2010

54. E. Castelli

"Mammography with synchrotron radiation: First clinical experience with phase-detection technique"

Radiology, vol. 259, pp. 684-694, 2011

55. A. C. Konstantinidis , A. Olivo and R. D. Speller

"Technical note: Modification of the standard gain correction algorithm to compensate for the number of used reference flat frames in detector performance studies"

Med. Phys., vol. 38, pp. 6683-6687, 2011

56. E. Samei , M. J. Flynn and D. A. Reimann

"A method for measuring the presampled MTF of digital radiographic systems using an edge test device"

Med. Phys., vol. 25, pp. 102-113, 1998

57. Medical Electrical Equipment-Characteristics of Digital X-Ray Imaging Devices-Part 1-2: Determination of the Detective Quantum Efficiency-Mammography Detectors IEC, International Electrotechnical Commission

2005

58. E. Buhr , S. Gunther-Kohfahl and U. Neitzel

"Accuracy of a simple method for deriving the presampled modulation transfer function of a digital radiographic system from an edge image"

Med. Phys., vol. 30, pp. 2323-2331, 2003

59. [online] Available:

http://www.alfa.com.

60. J. T. Dobbins III J. Beutel , H. L. Kundel and R. L. Van Metter

Handbook of Medical Imaging Physics and Psychophysics

vol. 1, pp. 190-203, 2000, SPIE

61. H. E. Johns and J. R. Cunningham

The Physics of Radiology

pp. 261-266, 1983, Charles C Thomas

62. J. M. Boone

"Spectral modeling and compilation of quantum fluence in radiography and mammography"

Proc. SPIE, vol. 3336, pp. 592-601, 1998

63. E. Samei and M. Flynn

"An experimental comparison of detector performance for computed radiography systems"

Med. Phys., vol. 29, pp. 447-459, 2002

64. Mammography: Assessment of Image Quality

vol. 9, pp. 33-51, 2009, Oxford Univ. Press 
65. N. W. Marshall

"Detective quantum efficiency measured as a function of energy for two full-field digital mammography systems"

Phys. Med. Biol., vol. 54, pp. 2845-2861, 2009

66. I. A. Cunningham J. Beutel , H. L. Kundel and R. L. Van Metter

Handbook of Medical Imaging Physics and Psychophysics

vol. 1, pp. 123-126, 2000, SPIE

67. R. Gwin and R. B. Murray

"Scintillation process in CsI(T1). I. Comparison with activator saturation model"

Phys. Rev., vol. 131, pp. 501-508, 1963

68. D. W. Aitken , B. L. Beron , G. Yenicay and H. R. Zulliger

"The fluorescent response of $\mathrm{NaI}(\mathrm{Tl}), \mathrm{CsI}(\mathrm{Tl}), \mathrm{CsI}(\mathrm{Na})$ and $\mathrm{CaF}(\mathrm{Eu})$ to X-rays and low energy gamma rays"

IEEE Trans. Nucl. Sci., vol. 14, pp. 468-477, 1967

69. W. Mengesha, T. D. Taulbee, B. D. Rooney and J. D. Valentine

"Light yield nonproportionality of CsI(Tl), CsI(Na), and YAP"

IEEE Trans. Nucl. Sci., vol. 45, pp. 456-461, 1998

70. C. D. Arvanitis , S. E. Bohndiek, J. Blakesley, A. Olivo and R. D. Speller

"Signal and noise transfer properties of CMOS based active pixel flat panel imager coupled to structured CsI:Tl"

Med. Phys., vol. 36, pp. 116-126, 2009

71. K. A. Fetterly and N. J. Hangiandreou

"Effects of X-ray spectra on the DQE of a computed radiography system"

Med. Phys., vol. 28, pp. 241-249, 2001

72. N. W. Marshall

"A comparison between objective and subjective image quality measurements for a full field digital mammography system"

Phys. Med. Biol., vol. 51, pp. 2441-2463, 2006

73. D. B. Williams

"Digital radiography image quality: Image acquisition"

J. Amer. College Radiol., vol. 4, pp. 371-388, 2007

74. A. C. Konstantinidis , A. Olivo and R. D. Speller

"Technical note: Further development of a resolution modification routine for the simulation of the modulation transfer function (MTF) of digital x-ray detectors"

Med. Phys., vol. 38, pp. 5916-5920, 2011

75. N. W. Marshall , P. Monnin , H. Bosmans , F. O. Bochud and F. R. Verdun

"Image quality assessment in digital mammography: Part I. Technical characterization of the systems"Phys. Med. Biol., vol. 56, pp. 4201-4220,

76. M. Skarpathiotakis

Contrast digital mammography 2002

77. F. Diekmann and S. Diekmann

"The future of breast cancer"

Diagn. Breast Care, vol. 3, pp. 384-387, 2008 